\title{
Alpha Power Transformed Weibull-G Family of Distributions: Theory and Applications
}

\author{
I. Elbatal ${ }^{1}$, M. Elgarhy ${ }^{2}$, B. M. Golam Kibria ${ }^{3,}$, ,(1) \\ ${ }^{1}$ Department of Mathematics and Statistics, College of Science Imam Mohammad Ibn Saud Islamic University (IMSIU), Riyadh, 11432, Saudi Arabia \\ ${ }^{2}$ The Higher Institute of Commercial Sciences, Al mahalla Al kubra, 31951, Algarbia, Egypt \\ ${ }^{3}$ Department of Mathematics and Statistics, Florida International University, Miami, FL, 33199, USA
}

\section{ARTICLE INFO}

Article History

Received 01 Jul 2020

Accepted 19 Feb 2021

Keywords

Application

Exponential distribution

Family of distribution

Generating function

Lindley distribution

Maximum likelihood estimation

Weibull distribution

Alpha power transformed

Distributions

Moments

Weibull -G family

MSC 2010 No: Primary: 62E05; Secondary: 60E99

\section{ABSTRACT}

This paper considers three special cases: Exponential, Rayleigh and Lindley of a family of generalized distributions, called alpha power Weibull G (APW-G) family. Some essential and valuable statistical properties of the family of distributions are obtained. The proposed distributions are very flexible and can be used to model data with decreasing, increasing or bathtub-shaped hazard rates. Model parameters estimation and a simulation study is carried out to evaluate the behaviors of the parameters. We consider two worthwhile data sets to illustrate the finding of the paper.

(C) 2021 The Authors. Published by Atlantis Press B.V. This is an open access article distributed under the CC BY-NC 4.0 license (http://creativecommons.org/licenses/by-nc/4.0/).

\section{INTRODUCTION}

There are many classical distributions that have been developed and discussed their statistical properties and applications for various discipline in past several years. However, most of them are not suitable to model the heavy-tailed data sets (Ahmad et al. [1]). As a results, for application purposes, it is required to have the extended forms of these distributions for various fields. So, it becomes great interest among researchers to generate new univariate distributions, by adding more shape parameters to a standard distribution. The generated new distributions are more flexible in modelling data in practice. Some famous generators families are, Exponentiated -Weibull -generator (Elgarhy et al. [2]), the Marshall-Olkin - G family by Marshal and Olkin [3], beta - G family by Eugene et al. [4], the generalized odd log logistic -G by Cordeiro et al. [5], the generalized transmuted- G by Nofal et al. [6], the odd Lindley- G family by Gomes et al. [7], a new extended alpha power transformed (APT)- G by Ahmad et al. [8], a new APT- G by Elbatal et al. [9], a new power Topp-Leone- G by Bantan et al. [10], type II general inverse exponential- G by Jamal et al. [11], Truncated Inverted Kumaraswamy- G by Bantan et al. [12], the exponentiated truncated inverse Weibull-G by Almarashi et al. [13], truncated Cauchy power- G by Aldahlan et al. [14] and type II power Topp-Leone- G by Bantan et al. [15], among others.

For an arbitrary baseline cumulative distribution function (cdf) $G(x)$, Mahadavi and Kundu [16] suggested APT family of distributions by introducing an extra parameter $\alpha$ to a distribution $\mathrm{G}$ to get a more flexible family. The cdf and probability density function (pdf) of APT family are provided, respectively, by (A1) and (A2) in Appendix A.

Several properties of the APT family have been investigated by many authors. See (Dey et al. [17,18], Hassan et al. [19,20] and Ihtisham et al. [21]), etc.

\footnotetext{
*Corresponding author. Email: kibriag@fiu.edu
} 
Proposed the Weibull- $G(W-G)$ family of distribution and defined the cdf and pdf of their Weibull- $G$ family mentioned in (A3) and (A4) in Appendix.

The Weibull distribution is a well-known lifetime distribution and it has extensive applications in reliability theory, specifically, it is has been used for analyzing biological, medical, quality control and engineering data sets. However, when the hazard rates are bathtub, upside down bathtub or bimodal shapes, it does not work that well. As a result, in recent years, researchers are motivated to develop numerous generalizations and extensions of the Weibull distribution to model different kind of data. Therefore, the objective of this paper is to introduce a new extended generator called alpha power transformed Weibull-G $(A P T W-G)$ family based on combined the alpha power transformed generator with the Weibull-G family of distributions. It is expected that the proposed distribution will be more flexible and will perform better than some existing probability distributions to model life testing data. We will provide three submodels of this family by taking the baseline distributions as exponential, Rayleigh and Lindley, in the hope that they will perform better than some existing distributions. We will provide the maximum likelihood estimation (MLE) technique for the estimations of the parameters.

The organization of this paper as follows. We provide the new family called APTW - G family and its submodels in Section 2. We also demonstrate that the $(A P T W-G)$ density is given by a linear combination of exponentiated - G (exp - G) densities. Some useful statistical properties of $A P T W-G$ family are obtained in Section 3. The MLE technique will be discussed in Section 4. For illustration purposes, two real-life data are analyzed in Section 5. Finally, some concluding remarks are stated in Section 6.

\section{PROPOSED FAMILY OF DISTRIBUTIONS}

Here, will construct the new generator of $A P T W-G$ family of distributions based on the APT family and Weibull- G (W- G) family, by inserting (A3) into (A1). We have the cdf of new $A P T W-G$ family by

$$
F_{A P T W-G}(x ; \theta, \alpha, \delta)= \begin{cases}\frac{\alpha^{1-e^{-t_{i}^{\theta}}}-1}{\alpha-1} & \text { if } \alpha>0, \alpha \neq 1 \\ 1-e^{-t_{i}^{\theta}} & \text { if } \alpha=1\end{cases}
$$

The pdf of Equation (1) is given by

$$
f_{A P T W-G}(x ; \theta, \alpha, \delta)= \begin{cases}\log (\alpha) \theta g(x ; \delta) \frac{G(x ; \delta)^{\theta-1}}{(\alpha-1) \bar{G}(x ; \delta)^{\theta+1}} e^{-t_{i}^{\theta}} \alpha^{1-e^{-t_{i}^{\theta}}} & \text { if } \alpha>0, \alpha \neq 1 \\ \theta g(x ; \delta) \frac{G(x ; \delta)^{\theta-1}}{\bar{G}(x ; \delta)^{\theta+1}} e^{-t_{i}^{\theta}} & \text { if } \alpha=1\end{cases}
$$

where $t_{i}=\frac{G(x ; \delta)}{\bar{G}(x ; \delta)}$. From now and onward, we will write simply $G(x ; \delta)=G(x), g(x ; \delta)=g(x), F_{A P T W-G}(x ; \theta, \alpha, \delta)=F_{A P T W-G}(x)$ and $f_{A P W-G}(x ; \theta, \alpha, \delta)=f_{A P W-G}(x)$. A random variable $X$ with pdf $(2)$ is denoted by $X \sim A P T W-G(\theta, \alpha, \delta)$.

The survival function $R_{A P W-G}(x)$ hazard rate function $h_{A P W-G}(x)$ and cumulative hazard rate function $H_{A P W-G}(x)$ are given respectively as

$$
\begin{aligned}
& R_{A P T W-G}(x ; \theta, \alpha, \delta)= \begin{cases}\frac{\alpha-\alpha^{1-e^{-t_{i}^{\theta}}}}{\alpha-1} & \text { if } \alpha>0, \alpha \neq 1 \\
e^{-t_{i}^{\theta}} & \text { if } \alpha=1\end{cases} \\
& h_{A P T W-G}(x ; \theta, \alpha, \delta)= \begin{cases}\frac{\log (\alpha) \theta g(x ; \delta) G(x ; \delta)^{\theta-1}}{\left(\alpha-\alpha^{1-e^{-t_{i}^{\theta}}}\right) \bar{G}(x ; \delta)^{\theta+1}} e^{-t_{i}^{\theta}} \alpha^{1-e^{-t_{i}^{\theta}}} & \text { if } \alpha>0, \alpha \neq 1 \\
\theta g(x ; \delta) \frac{G(x ; \delta)^{\theta-1}}{\bar{G}(x ; \delta)^{\theta+1}} & \text { if } \alpha=1\end{cases}
\end{aligned}
$$

and

$$
H_{A P T W-G}(x ; \theta, \alpha, \delta)=\left\{\begin{array}{ll}
-\ln \left(\frac{\alpha-\alpha^{1-e^{-t_{i}^{\theta}}}}{\alpha-1}\right) & \text { if } \alpha>0, \alpha \neq 1 \\
t_{i}^{\theta} & \text { if } \alpha=1
\end{array} .\right.
$$




\subsection{Some Special of the APTW-G Models}

We consider three different cases of the $A P T W-G$ family of distributions by taking the baseline distributions as exponential, Rayleigh and Lindley in this section. The cdf and pdf of the baseline models are given in Table 1.

Alpha power transformed Weibull exponential (APTWE) distribution

$$
F_{A P T W E}(x)= \begin{cases}\frac{\alpha^{\left.1-e^{-\left(e^{\beta x}-1\right.}\right)^{\theta}}-1}{\alpha-1} & \text { if } \alpha>0, \alpha \neq 1 \\ 1-e^{-\left(e^{\beta x}-1\right)^{\theta}} & \text { if } \alpha=1\end{cases}
$$

The corresponding pdf is obtained as follows:

$$
f_{A P W E}(x)= \begin{cases}\log (\alpha) \theta \beta e^{-\beta x} \frac{\left(1-e^{-\beta x}\right)^{\theta-1}}{(\alpha-1)\left(e^{-\beta x}\right)^{\theta+1}} e^{-\left(e^{\beta x}-1\right)^{\theta}} \alpha^{1-e^{-\left(e^{\beta x}-1\right)^{\theta}}} & \text { if } \alpha>0, \alpha \neq 1 \\ \theta \beta e^{-\beta x} \frac{\left(1-e^{-\beta x}\right)^{\theta-1}}{\left(e^{-\beta x}\right)^{\theta+1}} e^{-\left(e^{\beta x}-1\right)^{\theta}} & \text { if } \alpha=1\end{cases}
$$

Alpha power transformed Weibull Rayleigh $(A P T W R)$ distribution

$$
F_{A P T W R}(x)=\left\{\begin{array}{l}
\frac{\alpha^{\left.1-e^{-\left(e^{\frac{\rho}{2} x^{2}}-1\right.}\right)^{\theta}}-1}{\alpha-1} \text { if } \alpha>0, \alpha \neq 1 \\
1-e^{-\left(e^{\frac{\rho}{2^{2}}-1}\right)^{\theta}} \text { if } \alpha=1
\end{array}\right.
$$

The corresponding pdf is given by

$$
f_{\text {APTWR }}(x)= \begin{cases}\log (\alpha) \theta \rho x e^{-\frac{\rho}{2} x^{2}} \frac{\left(1-e^{-\frac{\rho}{2} x^{2}}\right)^{\theta-1}}{(\alpha-1)\left(e^{-\frac{\rho}{2} x^{2}}\right)^{\theta+1}} e^{-\left(e^{\frac{\rho}{2} x^{2}}-1\right)^{\theta}} \alpha^{\left.1-e^{-\left(e^{\frac{\rho}{x^{2}}}-1\right.}\right)^{\theta}} & \text { if } \alpha>0, \alpha \neq 1 \\ \theta \rho x e^{-\frac{\rho}{2} x^{2}} \frac{\left(1-e^{-\frac{\rho}{2} x^{2}}\right)^{\theta-1}}{\left.\left(e^{-\frac{\rho}{2} x^{2}}\right)^{-\left(e^{\frac{\rho}{2} x^{2}}-1\right.}\right)^{\theta}} & \text { if } \alpha=1\end{cases}
$$

Table 1 List of few special members of the alpha power Weibull-G (APTW-G) family distribution.

\begin{tabular}{lccc}
\hline Model & $C d f: G(x ; \delta)$ & $p d f: g(x ; \delta)$ & $\frac{G(x ; \delta)}{\bar{G}(x ; \delta)}$ \\
Exponential & $1-e^{-\beta x}$ & $\beta e^{-\beta x}$ & $\left(e^{\beta x}-1\right)$ \\
Rayleigh & $1-e^{-\frac{\beta}{2} x^{2}}$ & $\beta x e^{-\frac{\beta}{2} x^{2}}$ & $\left(e^{\frac{\beta}{2} x^{2}}-1\right)$ \\
Lindley & $1-\left(1+\frac{a x}{1+a}\right) e^{-a x}$ & $\frac{a^{2}}{1+a}(1+x) e^{-a x}$ & $\left(\frac{e^{a x}}{\left(1+\frac{a x}{1+a}\right)}-1\right)$ \\
\hline
\end{tabular}


Alpha-power transformed Weibull Lindley $(A P T W L)$ distribution

$$
F_{A P T W L}(x)= \begin{cases}\frac{\alpha^{1-e}\left(\frac{e^{a x}}{\left(1+\frac{a x}{1+a}\right)^{-1}}\right)^{\theta}-1}{\alpha-1} & \text { if } \alpha>0, \alpha \neq 1 \\ -\left(\frac{e^{a x}}{\left(1+\frac{a x}{1+a}\right)}-1\right)^{\theta} & \text { if } \alpha=1\end{cases}
$$

The corresponding pdf is obtained as follows:

$$
f_{\text {APTWL }}(x)= \begin{cases}\log (\alpha) \theta \frac{a^{2}}{1+a}(1+x) e^{-a x} \frac{\left(1-\left(1+\frac{a x}{1+a}\right) e^{-a x}\right)^{\theta-1}}{(\alpha-1)\left(\left(1+\frac{a x}{1+a}\right) e^{-a x}\right)^{\theta+1}} e^{-t_{i}^{\theta} \alpha^{1-e}}-\left(\frac{e^{a x}}{\left(1+\frac{a x}{1+a}\right)^{-1}}\right)^{\theta} & \text { if } \alpha>0, \alpha \neq 1 \\ \theta \frac{a^{2}}{1+a}(1+x) e^{-a x} \frac{\left(1-\left(1+\frac{a x}{1+a}\right) e^{-a x}\right)^{\theta-1}}{\left(\left(1+\frac{a x}{1+a}\right) e^{-a x}\right)^{\theta+1}} e^{\left(\frac{e^{a x}}{\left(1+\frac{a x}{1+a}\right)}-1\right)^{\theta}} & \text { if } \alpha=1\end{cases}
$$

The pdf (left panel) and hazard rate function (right panel) for different set of parameters, of APTWE, APTWR and APTWL are presented in Figures 1-3 respectively.

From Figures 1-3, we observed that the pdf of APTWE, APTWR and APTWL distributions can be decreasing, symmetry, unimodal and skewed to the right, depend on the values of the parameters. Also, the hazard rate function (HRF) of APTWE, APTWR and APTWL distributions can be increasing, $\mathrm{j}$ - shaped, $\mathrm{U}$ - shaped and decreasing depend on the values of the parameters.

\subsection{Expansion of the pdf of APTW-G Family}

We will derive the density expansion of APTW-G family of distributions in this section. Using the power series expansion

$$
\alpha^{Z}=\sum_{k=0}^{\infty} \frac{(\log \alpha)^{k}}{k !} Z^{k}, \alpha>0, \alpha \neq 1
$$
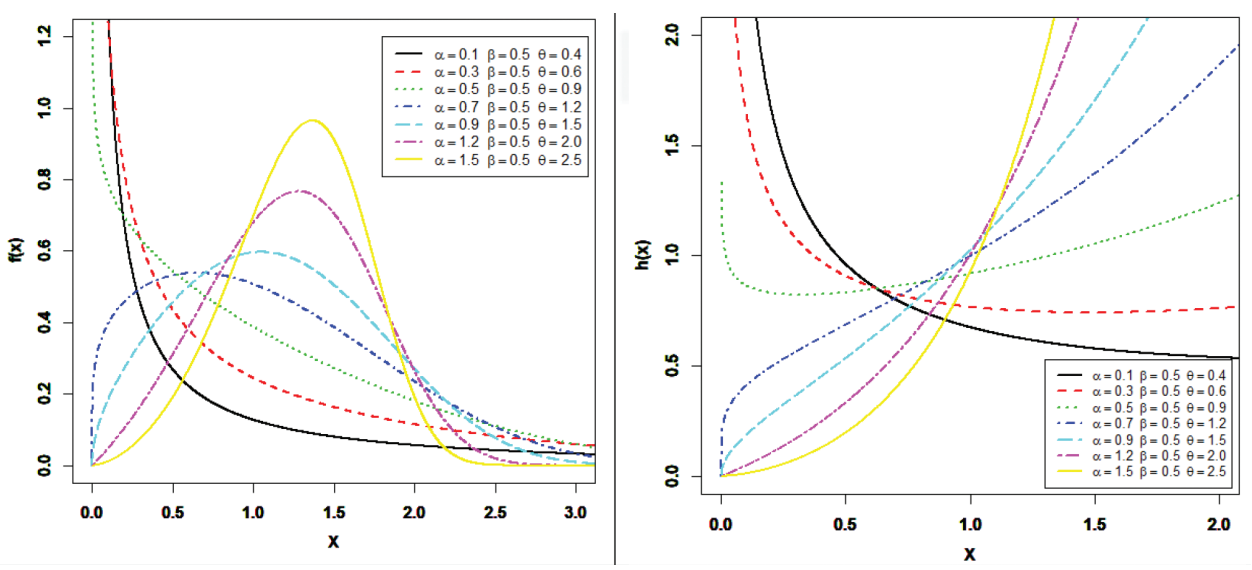

Figure 1 Plots of the pdf and hrf for different parameters of the alpha power transformed Weibull exponential (APTWE) distribution. 

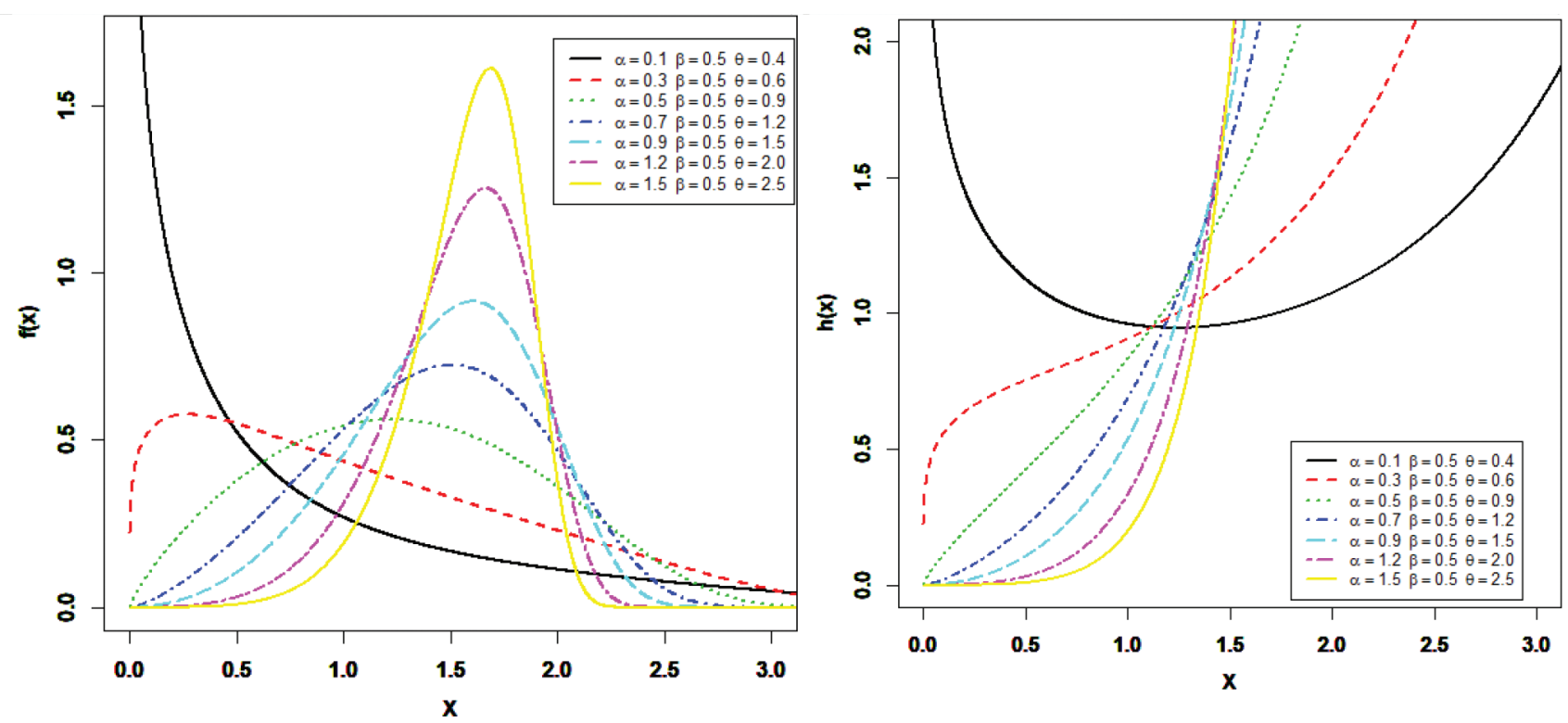

Figure 2 Plots of the pdf and hrf for different parameters of the alpha power transformed Weibull Rayleigh (APTWR) distribution.
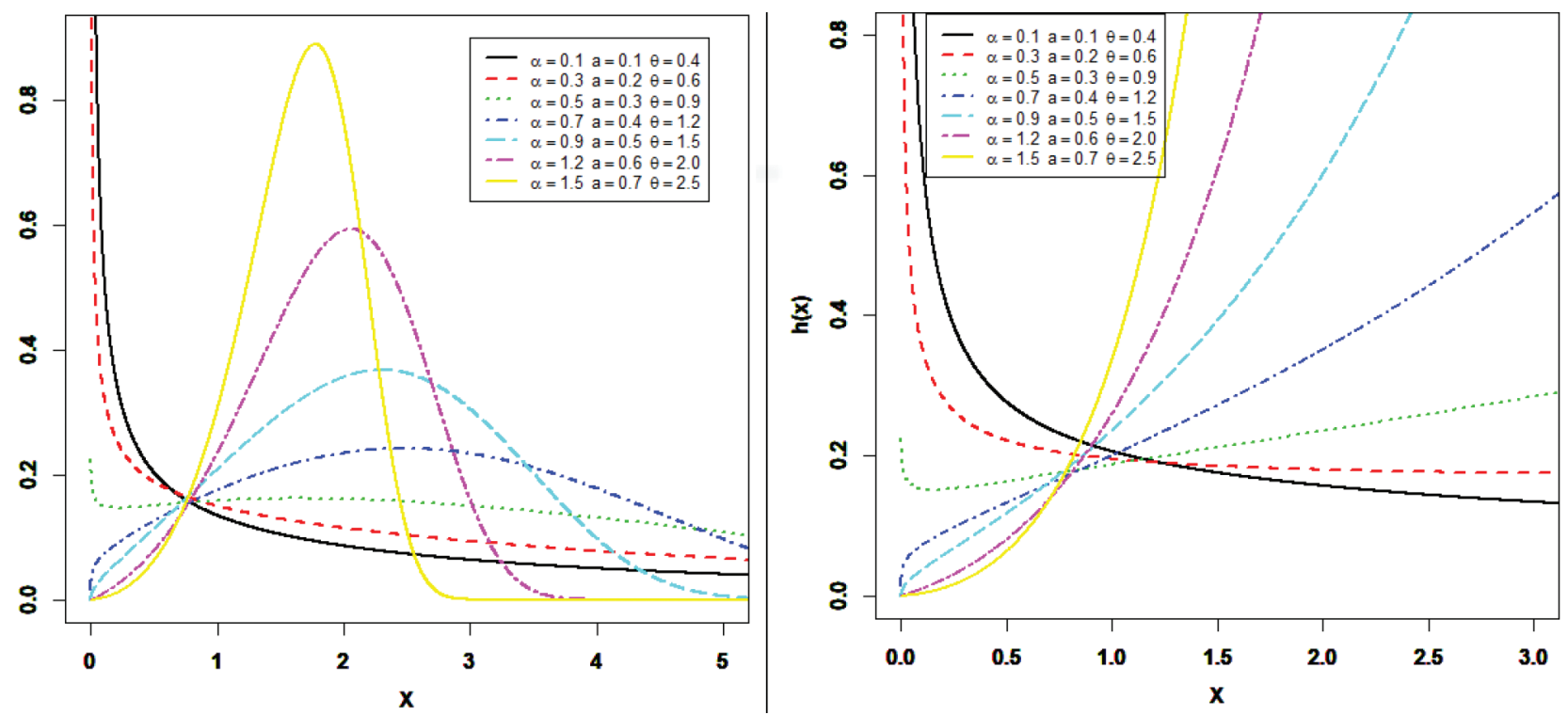

Figure 3 Plots of the pdf and hrf for different parameters of the alpha power transformed Weibull Lindley (APTWL) distribution.

the $A P T W-G$ family density can be given as

$$
f_{A P T W-G}(x)=\sum_{i=0}^{\infty} \frac{(\log \alpha)^{i+1} \theta g(x ; \delta) G(x ; \delta)^{\theta-1}}{i !(\alpha-1) \bar{G}(x ; \delta)^{\theta+1}} e^{-\left[\frac{G(x ; \delta)}{\bar{G}(x ; \delta)}\right]^{\theta}}\left[1-e^{-\left[\frac{G(x ; \delta)}{\bar{G}(x ; \delta)}\right]^{\theta}}\right]^{i}
$$

but

$$
\left[1-e^{-\left[\frac{G(x ; \delta)}{\bar{G}(x ; \delta)}\right]^{\theta}}\right]^{i}=\sum_{j=0}^{\infty}(-1)^{j}\left(\begin{array}{l}
i \\
j
\end{array}\right) \exp \left\{-j\left[\frac{G(x ; \delta)}{\bar{G}(x ; \delta)}\right]^{\theta}\right\},
$$


applying (5) to the last term in (4) gives

$$
f_{A P T W-G}(x)=\sum_{i, j=0}^{\infty} \frac{(-1)^{j}(\log \alpha)^{i+1}\left(\begin{array}{l}
i \\
j
\end{array}\right) \theta g(x ; \delta) G(x ; \delta)^{\theta-1}}{i !(\alpha-1) \bar{G}(x ; \delta)^{\theta+1}} e^{-(\mathrm{j}+1)\left[\frac{G(x ; \delta)}{\bar{G}(x ; \delta)}\right]^{\theta}}
$$

Using the power series of the exponential function, we obtain

$$
\exp \left\{-(j+1)\left[\frac{G(x ; \delta)}{\bar{G}(x ; \delta)}\right]^{\theta}\right\}=\sum_{k=0}^{\infty} \frac{(-1)^{k}[(j+1)]^{k}}{k !}\left[\frac{G(x ; \delta)}{\bar{G}(x ; \delta)}\right]^{\theta k}
$$

inserting this expansion in (4) we get

$$
f_{A P T W-G}(x)=\sum_{i, j, k=0}^{\infty} \frac{(-1)^{j+k}(\log \alpha)^{i+1}[(j+1)]^{k}\left(\begin{array}{c}
i \\
j
\end{array}\right) \theta}{i ! k !(\alpha-1)} \times \frac{g(x ; \delta) G(x ; \delta)^{\theta(k+1)-1}}{\bar{G}(x ; \delta)^{\theta(k+1)+1}},
$$

Furthermore, using the general binomial expansion

$$
(1-z)^{-a}=\sum_{m=0}^{\infty} \frac{(a)_{m}}{m !} z^{m}
$$

where $(a)_{m}=\frac{\Gamma(a+m)}{\Gamma(a)}=a(a+1) \ldots(a+m-1)$ is the ascending factorial, $|z|<1$ and $\Gamma($.$) is a gamma function, we can write$

$$
[1-G(x ; \delta)]^{-(\theta(k+1)+1)}=\sum_{d=0}^{\infty} \frac{(\theta(k+1)+1)_{d}}{d !} G(x ; \delta)^{d}
$$

so, the pdf of $A P T W-G$ can be expressed as an infinite linear combination of exp-G density functions as follows:

$$
f_{\text {APTW-G }}(x)=\sum_{k, d=0}^{\infty} \varpi_{k, d} \pi_{(k+1) \theta+d}(x)
$$

where

$$
\varpi_{k, d}=\sum_{i, j=0}^{\infty} \frac{(-1)^{j+k}(\log \alpha)^{i+1} \theta[\lambda(j+1)]^{k}\left(\begin{array}{l}
i \\
j
\end{array}\right)(\theta(k+1)+1)_{d}}{i ! k ! d !(\alpha-1)(d+\theta(k+1))}
$$

and

$$
\pi_{\xi}(x)=\xi g(x ; \delta) G(x ; \delta)^{\xi-1}
$$

is the exp-G pdf with power parameter $\xi$. Equation (9) indicates that the density function of random variable $X$ can be expressed as an infinite mixture of the exp-G densities with parameter $(k+1) \theta+d$, therefore the APTW $-G$ density can be considered as a linear combination of the exp-G densities. Thus, a lot of interesting statistical and mathematical properties of the $A P T W-G$ family of distributions come directly from those of exp-G distribution. Likewise, the cdf of the $A P T W-G$ family of distributions can be written as a linear combination of exp-G cdfs which is given by

$$
F_{A P T W-G}(x)=\sum_{k, d=0}^{\infty} \varpi_{k, d} \prod_{(k+1) \theta+d}(x)
$$

where $\Pi_{(k+1) \theta+d}(x)$ is the exp-G cdf with power parameter $(k+1) \theta+d$. 


\section{SOME STATISTICAL PROPERTIES}

Most of the formulae or mathematical expressions of this paper can easily be derived using Mathematica and Maple because of their ability to deal with complex expressions.

\subsection{Quantile Function}

The $A P T W-G$ quantile function $(\mathrm{QF})$, say $x=Q(u)$ can be obtained by inverting (1), we have

$$
x=Q(u)=G^{-1}\left[\frac{\left(-\log \left(1-\frac{\log (1+u(\alpha-1)}{\log (\alpha)}\right)\right)^{\frac{1}{\theta}}}{1+\left(-\log \left(1-\frac{\log (1+u(\alpha-1)}{\log (\alpha)}\right)\right)^{\frac{1}{\theta}}}\right] .
$$

One can generates $X$ easily by taking $u$ as a uniform random variable in $(0,1)$.

\subsection{Moments}

The $r_{\text {th }}$ moment of $X$ can be obtained from Equation (9) as follows:

$$
\mu_{r}^{\prime}=E\left(X^{r}\right)=\int_{0}^{\infty} x^{r} f_{A P T W-G}(x) d x=\sum_{k, d=0}^{\infty} \varpi_{k, d} E\left(Z_{(k+1) \theta+d}^{r}\right)
$$

where $E\left(Z_{(k+1) \theta+d}^{r}\right)$ denotes the $r_{t h}$ moment of $Z_{(k+1) \theta+d}$ which follows the exp - G random variable with power parameter $(k+1) \theta+d$. Another formula for the $r_{t h}$ moment follows from (9) as:

$$
\mu_{r}^{\prime}=E\left(X^{r}\right)=\sum_{k, d=0}^{\infty} \varpi_{k, d} E\left(Z_{(k+1) \theta+d}^{r}\right)
$$

where $E\left(Z_{\xi}^{r}\right)=\xi \int_{-\infty}^{\infty} x^{r} g(x ; \delta) G(x ; \delta)^{\xi-1}, \xi>0$ can be obtained using numerical computation in terms of the baseline $Q F$, i.e., $Q_{G}(u)=$ $G^{-1}(u)$ as $E\left(Z_{\xi}^{r}\right)=\xi \int_{0}^{1} u^{\xi-1} Q_{G}(u)^{r} d u$. Also the $n_{t h}$ central moment of $X$, say $\Lambda_{n}$ is given by

$$
\begin{aligned}
\Lambda_{n} & =E\left(X-\mu_{1}^{\prime}\right)^{n}=\sum_{r=0}^{n}\left(\begin{array}{c}
n \\
r
\end{array}\right)\left(-\mu_{1}^{\prime}\right)^{n-r} E\left(X^{r}\right) \\
& =\sum_{r=0}^{n} \sum_{k, d=0}^{\infty}\left(\begin{array}{l}
n \\
r
\end{array}\right)\left(-\mu_{1}^{\prime}\right)^{n-r} \varpi_{k, d} E\left(Z_{(k+1) \theta+d}^{r}\right) .
\end{aligned}
$$

\subsection{Moment Generating Function}

We display two different expressions for the moment generating function. The first one can be calculated using (9) as follows:

$$
M_{X}(t)=E\left(e^{t X}\right)=\sum_{k, d=0}^{\infty} \varpi_{k, d} M_{(k+1) \theta+d}(t),
$$

where $M_{(k+1) \theta+d}(t)$ is the moment generating function of $Z_{(k+1) \theta+d}$. Consequently, we can be easily determined $M_{X}(t)$ from the exp- $\mathrm{G}$ generating function. The second expression for the $M_{X}(t)$ follows from (9) as:

$$
M_{X}(t)=E\left(e^{t X}\right)=\sum_{k, d=0}^{\infty} \varpi_{k, d} \varepsilon(t,(k+1) \theta+d)
$$


where

$$
\varepsilon_{p}(t)=p \int_{0}^{1} u^{p-1} e^{t Q_{G}(u)} d u
$$

which will be computed numerically from the baseline QF, i.e., $Q_{G}(u)=G^{-1}(u)$.

\subsection{Conditional Moments}

Here, we will discuss about the conditional moments, which are important for prediction in lifetime models. The $s_{t h}$ incomplete moments of $X$ defined by $\vartheta_{s}(t)$ for any real $s>0$ can be expressed from (9) as

$$
\vartheta_{s}(t)=\int_{-\infty}^{t} x^{s} f(x) d x=\sum_{k, d=0}^{\infty} \varpi_{k, d} \int_{-\infty}^{t} x^{s} \pi_{(k+1) \theta+d}(x) d x
$$

where the integral in (13) represents the $s_{t h}$ incomplete moments of $Z_{(k+1) \theta+d}$.

\subsection{Mean Deviations, Bonferroni and Lorenz Curves}

The mean deviations about the mean $\mu=E(X)$ and the mean deviations about the median $M$ are defined respectively by

$$
\delta_{1}(x)=E\left|X-\mu_{1}^{\prime}\right|=2 \mu_{1}^{\prime} F\left(\mu_{1}^{\prime}\right)-2 \vartheta_{1}\left(\mu_{1}^{\prime}\right)
$$

and

$$
\delta_{2}(x)=E|X-M|=\mu_{1}^{\prime}-2 \vartheta_{1}(M)
$$

where $\mu_{1}^{\prime}=E(X), M=$ median $(X)=Q\left(\frac{1}{2}\right), F\left(\mu_{1}^{\prime}\right)$ is easily computed from (1) and $\vartheta_{1}(t)$ is the first complete moment given by (19) with $s=1$. Now we provide two ways to determine $\delta_{1}(x)$ and $\delta_{2}(x)$. First, using Equation (9), a general equation for $\vartheta_{1}(t)$ can be obtained as follows:

$$
\vartheta_{1}(t)=\sum_{k, d=0}^{\infty} \varpi_{k, d} \Omega_{(k+1) \theta+d}(t)
$$

where $\Omega_{(k+1) \theta+d}(t)=\int_{-\infty}^{t} x \pi_{(k+1) \theta+d}(x) d x$ is the first complete moment of the exp-G distribution. A second formula for $\vartheta_{1}(t)$ is given by

$$
\vartheta_{1}(t)=\sum_{k, d=0}^{\infty} \varpi_{k, d} \Delta_{(k+1) \theta+d}(t)
$$

where

$$
\Delta_{(k+1) \theta+d}(t)=((k+1) \theta+d) \int_{0}^{G(t)} Q_{G}(u) u^{(k+1) \theta+d} d u
$$

can be calculated numerically.

\section{MAXIMUM LIKELIHOOD ESTIMATION}

Let $x_{1}, \ldots, x_{n}$ be a random sample of size $n$ from the $A P T W-G$ distribution given by (2). Let $\left.\psi=(\theta, \alpha, \delta)\right)^{T}$ be $p \times 1$ vector of parameters. The total $\log$-likelihood function for $\psi$ is given by

$$
\begin{aligned}
L_{n}= & n \log (\log (\alpha))-n \log (\alpha-1)+n \log (\theta)+\sum_{i=1}^{n} \log g\left(x_{i} ; \delta\right)+(\theta-1) \sum_{i=1}^{n} \log G\left(x_{i} ; \delta\right) \\
& -(\theta+1) \sum_{i=1}^{n} \log \bar{G}\left(x_{i} ; \delta\right)-\sum_{i=1}^{n} t_{i}^{\theta}+\sum_{i=1}^{n}\left(1-e^{-t_{i}^{\theta}}\right) \log (\alpha)
\end{aligned}
$$


Using R-language, the log-likelihood can be by solving the nonlinear likelihood equations obtained by differentiating (16). The associated components of the score function $U_{n}(\psi)=\left[\frac{\partial L_{n}}{\partial \theta}, \frac{\partial L_{n}}{\partial \alpha}, \frac{\partial L_{n}}{\partial \delta}\right]^{T}$ are

$$
\begin{aligned}
\frac{\partial L_{n}}{\partial \theta}= & \frac{n}{\theta}+\sum_{i=1}^{n} \log G\left(x_{i} ; \delta\right)-\sum_{i=1}^{n} \log \bar{G}\left(x_{i} ; \delta\right) \\
& -\sum_{i=1}^{n} t_{i}^{\theta} \log \left(t_{i}\right)+\log (\alpha) \sum_{i=1}^{n} t_{i}^{\theta} \log \left(t_{i}\right) e^{-t_{i}^{\theta}} \\
\frac{\partial L_{n}}{\partial \alpha}= & \frac{n}{\alpha \log (\alpha)}-\frac{n}{\alpha-1}+\frac{1}{\alpha} \sum_{i=1}^{n}\left(1-e^{-t_{i}^{\theta}}\right)
\end{aligned}
$$

and

$$
\begin{aligned}
\frac{\partial L_{n}}{\partial \delta_{k}}= & \sum_{i=1}^{n} \frac{{ }^{\prime}\left(x_{i} ; \delta\right)}{g\left(x_{i} ; \delta\right)}+(\theta-1) \sum_{i=1}^{n} \frac{{ }^{\prime}}{G\left(x_{i} ; \delta\right)} \frac{1}{G\left(x_{i} ; \delta\right)}-(\theta+1) \sum_{i=1}^{n} \frac{1}{\bar{G}\left(x_{i} ; \delta\right)} \times \frac{\partial \bar{G}(x ; \delta)}{\partial \delta_{k}} \\
& -\theta \sum_{i=1}^{n} t_{i}^{\theta-1} \frac{\partial t_{i}}{\partial \delta_{k}}+\log (\alpha) \theta \sum_{i=1}^{n} e^{-t_{i}^{\theta}} t_{i}^{\theta-1} \frac{\partial t_{i}}{\partial \delta_{k}}
\end{aligned}
$$

where $g^{\prime}\left(x_{i} ; \delta\right)=\frac{\partial g\left(x_{i} ; \delta\right)}{\partial \delta_{k}}, G^{\prime}\left(x_{i} ; \delta\right)=\frac{\partial G\left(x_{i} ; \delta\right)}{\partial \delta_{k}}$, and $\delta_{k}$ is the $k^{\text {th }}$ element of the vector of parameters $\delta$. The MLE of $\psi$, say $\hat{\psi}$, is obtained by solving the nonlinear system $U_{n}(\psi)=0$. Statistical software can be used to solve numerically these equations via iterative methods.

To evaluate the performance of the Maximum Likelihood (ML) of APTWE, a Monte Carlo simulation is consider. The process is arranged as follows:

- We generate random samples from APTWE model.

- The number of Monte Carlo replications was made 1000 times each with sample sizes 30, 50 and 100.

- Selected values for the parameters are choice as reported in Tables 2-4.

- Formulas used for calculating mean square error (MSE), lower bound (LB), average length (AL) and upper bound (UB) of 90\% and 95\% are calculated.

- Step (iii) is also repeated for the other parameters.

All calculations in this section getting by using via Mathematica 9.

The simulation results for different sample sizes (30, 50 and 100) and different values of parameters $(\alpha=0.5, \beta=0.5, \theta=0.5), \alpha=0.8, \beta=$ $0.5, \theta=0.5$, and $\alpha=0.8, \beta=0.8, \theta=0.5$ are presented in Tables $2-4$ respectively. These tables evidence the estimation consistency of the estimators.

\begin{tabular}{|c|c|c|c|c|c|c|c|c|}
\hline \multirow[b]{2}{*}{$n$} & \multirow[b]{2}{*}{ MLE } & \multirow[b]{2}{*}{ MSE } & \multicolumn{3}{|c|}{$90 \%$} & \multicolumn{3}{|c|}{$95 \%$} \\
\hline & & & LB & UB & AL & LB & UB & AL \\
\hline \multirow{4}{*}{30} & 0.591 & 0.031 & 0.194 & 0.989 & 0.795 & 0.118 & 1.065 & 0.947 \\
\hline & 0.627 & 0.222 & -0.361 & 1.615 & 1.977 & -0.551 & 1.805 & 2.355 \\
\hline & 0.614 & 0.073 & 0.219 & 1.008 & 0.79 & 0.143 & 1.084 & 0.941 \\
\hline & 0.476 & 0.007 & 0.293 & 0.659 & 0.366 & 0.258 & 0.694 & 0.436 \\
\hline \multirow[t]{2}{*}{50} & 0.434 & 0.057 & -0.002 & 0.871 & 0.873 & -0.086 & 0.954 & 1.04 \\
\hline & 0.541 & 0.009 & 0.389 & 0.693 & 0.304 & 0.36 & 0.723 & 0.362 \\
\hline \multirow{3}{*}{100} & 0.506 & 0.004 & 0.385 & 0.628 & 0.242 & 0.362 & 0.651 & 0.289 \\
\hline & 0.572 & 0.043 & 0.232 & 0.911 & 0.679 & 0.167 & 0.976 & 0.809 \\
\hline & 0.488 & 0.003 & 0.412 & 0.564 & 0.151 & 0.398 & 0.578 & 0.18 \\
\hline
\end{tabular}

\section{APPLICATIONS}

For illustration purposes of the APTWE model, two real data sets are analyzed in this section. The first data was consider by Linhart and Zucchini [22], which signifies the failure times of air-conditioned system of an airplane. The second data set was consider by Aarset [23],

Table 2 Numerical results for APTWE model at $(\alpha=0.5, \beta=0.5, \theta=0.5)$. 
Table 3 Numerical results for APTWE model at $(\alpha=0.8, \beta=0.5, \theta=0.5)$.

\begin{tabular}{|c|c|c|c|c|c|c|c|c|}
\hline \multirow[b]{2}{*}{$n$} & \multirow[b]{2}{*}{ MLE } & \multirow[b]{2}{*}{ MSE } & \multicolumn{3}{|c|}{$90 \%$} & \multicolumn{3}{|c|}{$95 \%$} \\
\hline & & & LB & UB & AL & LB & UB & AL \\
\hline \multirow{3}{*}{30} & 1.2770 & 1.3630 & -0.1010 & 2.6550 & 2.7570 & -0.3650 & 2.9190 & 3.2850 \\
\hline & 0.9560 & 1.1530 & -0.5940 & 2.5070 & 3.1010 & -0.8910 & 2.8040 & 3.6950 \\
\hline & 0.4950 & 0.0050 & 0.3760 & 0.6150 & 0.2390 & 0.3530 & 0.6380 & 0.2850 \\
\hline \multirow{3}{*}{50} & 0.8890 & 0.0770 & 0.5180 & 1.2610 & 0.7420 & 0.4470 & 1.3320 & 0.8850 \\
\hline & 0.6290 & 0.1920 & 0.1060 & 1.1530 & 1.0470 & 0.0060 & 1.2530 & 1.2470 \\
\hline & 0.5140 & 0.0040 & 0.4200 & 0.6090 & 0.1890 & 0.4010 & 0.6270 & 0.2250 \\
\hline \multirow{3}{*}{100} & 0.7950 & 0.0060 & 0.5680 & 1.0230 & 0.4550 & 0.5240 & 1.0660 & 0.5420 \\
\hline & 0.4970 & 0.0130 & 0.2070 & 0.7880 & 0.5810 & 0.1510 & 0.8440 & 0.6920 \\
\hline & 0.5020 & 0.0010 & 0.4300 & 0.5740 & 0.1430 & 0.4170 & 0.5870 & 0.1710 \\
\hline
\end{tabular}

Table 4 Numerical results for APTWE model at $(\alpha=0.8, \beta=0.8, \theta=0.5)$.

\begin{tabular}{|c|c|c|c|c|c|c|c|c|}
\hline \multirow[b]{2}{*}{$n$} & \multirow[b]{2}{*}{ MLE } & \multirow[b]{2}{*}{ MSE } & \multicolumn{3}{|c|}{$90 \%$} & \multicolumn{3}{|c|}{$95 \%$} \\
\hline & & & LB & UB & $\mathrm{AL}$ & LB & UB & $\mathrm{AL}$ \\
\hline \multirow{3}{*}{30} & 0.929 & 0.115 & 0.365 & 1.492 & 1.128 & 0.257 & 1.6 & 1.343 \\
\hline & 1.012 & 0.298 & -0.157 & 2.181 & 2.338 & -0.381 & 2.405 & 2.786 \\
\hline & 0.493 & 0.004 & 0.367 & 0.619 & 0.251 & 0.343 & 0.643 & 0.3 \\
\hline \multirow{3}{*}{50} & 0.894 & 0.057 & 0.539 & 1.249 & 0.71 & 0.471 & 1.317 & 0.846 \\
\hline & 0.933 & 0.135 & 0.243 & 1.823 & 1.58 & 0.092 & 1.974 & 1.882 \\
\hline & 0.486 & 0.002 & 0.402 & 0.571 & 0.17 & 0.385 & 0.588 & 0.202 \\
\hline \multirow{3}{*}{100} & 0.849 & 0.026 & 0.603 & 1.096 & 0.493 & 0.555 & 1.143 & 0.588 \\
\hline & 0.759 & 0.085 & 0.321 & 1.197 & 0.877 & 0.237 & 1.281 & 1.044 \\
\hline & 0.509 & 0.001 & 0.452 & 0.605 & 0.153 & 0.438 & 0.619 & 0.182 \\
\hline
\end{tabular}

Table 5 Failures time data.

\begin{tabular}{ll}
\hline Data 1 & $23,261,87,7,120,14,62,47,225,71,246,21,42,20,5,12,120,11,3,14,71,11,14,11,16,90,1,16$, \\
& 52,95 \\
Data 2 & $0.1,0.2,1,1,1,1,1,2,3,6,7,11,12,18,18,18,18,18,21,32,36,40,45,46,47,50,55,60,63,63,67$, \\
& $67,67,67,72,75,79,82,82,83,84,84,84,85,85,85,85,85,86,86$ \\
\hline
\end{tabular}

which represents the failure times of 50 devices. All data sets are presented in Table 5. We fit the APTWE distribution and other four competing models namely; alpha power transformed Weibull inverse Lomax (APTIL) (ZeinEldein et al. [24]), alpha power transformed Lindley (APTL) (Dey et al. [17]), alpha power transformed exponential (APTE) (Mahadavi and Kundu [16]) distributions. The probability densities of these distributions is given by:

$$
\begin{gathered}
f_{\text {APTE }}(x)=\frac{\gamma \log \alpha e^{-\gamma x} \alpha^{1-e^{-\gamma x}}}{(\alpha-1)}, x, \alpha, \gamma>0, \alpha \neq 1, \\
f_{A P I L}(x)=\frac{a b(\log \alpha) x^{-2}\left(1+\frac{b}{x}\right)^{-a-1} \alpha\left(1+\frac{b}{x}\right)^{-a}}{(\alpha-1)}, x, \alpha, a, b>0, \alpha \neq 1,
\end{gathered}
$$

and

$$
f_{A P T L}(x)=\frac{\log \alpha}{\alpha-1} \frac{\beta \gamma^{2}(1+x) e^{-\gamma x} \alpha^{1-e^{-\gamma x}\left[1+\frac{\gamma x}{\gamma+1}\right]}}{\gamma+1}, x, \alpha, \gamma>0, \alpha \neq 1
$$

Figures 4 and 5 demonstrate the box and TTT plots for the two datasets respectively. Aarset (1987) proposed that the hrf is decreasing or increasing if the graph of TTT plot is a convex or concave. The hrf is U-shaped (bathtub) if the TTT plot is firstly convex and then concave.

The ML estimates along with their standard error (SE) of the model parameters for the first and second sets are provided in Tables 6 and 7 respectively. In the same tables, the analytical measures including; minus log-likelihood(-log L) Kolmogorov-Smirnov (KS) test statistic, Akaike information criterion (AIC), corrected Akaike information criterion (CAIC), Bayesian information criterion (BIC) and Hannan-Quinn information criterion (HQIC) are presented. 
From Tables 6 and 7, it is evident that the proposed APWE distribution provides the overall best fit and therefore could be chosen as the more adequate model among APTWE, APTIL, APTL and APTE models. We also plotted epdf, ecdf, esf and PP plots in Figures 6 and 7 for first and second data sets respectively.
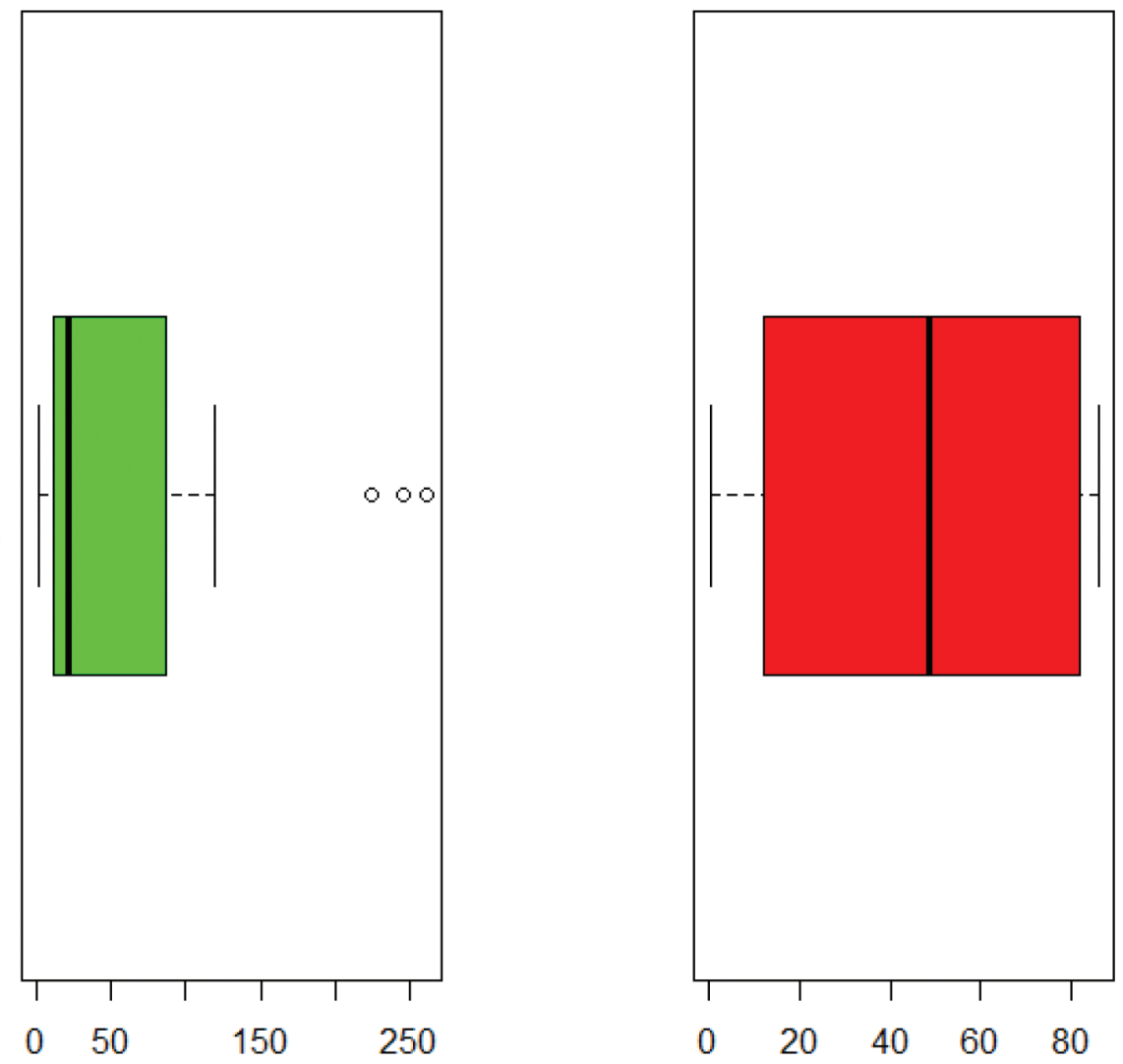

Figure 4 Box plots of both data sets.

Table 6 Analytical results of the APTWE model and other competing models for the first data set.

\begin{tabular}{|c|c|c|c|c|c|c|c|}
\hline Model & ML Estimates (SE) & $-\log L$ & AIC & BIC & CAIC & HQIC & KS \\
\hline APTWE & $\hat{\alpha}=0.051(0.107) \hat{\beta}=4.941 \times 10^{-3}(0.00251) \hat{\theta}=0.85(0.13)$ & 176.788 & 359.576 & 358.007 & 360.499 & 360.92 & 0.1497 \\
\hline APTIL & $\hat{\alpha}=39.286(163.336) \hat{a}=1.705(2.581) \hat{b}=3.5(4.478)$ & 177.573 & 361.146 & 359.578 & 361.668 & 362.491 & 0.2007 \\
\hline APTL & $\hat{\alpha}=0.1(0.104) \hat{\gamma}=0.024\left(5.127 \times 10^{-3}\right)$ & 183.415 & 370.83 & 369.784 & 371.274 & 371.727 & 0.2803 \\
\hline APTE & $\hat{\alpha}=8.688 \times 10^{-10}\left(5.698 \times 10^{-8}\right) \hat{\gamma}=8.536 \times 10^{-4}\left(2.844 \times 10^{-3}\right)$ & 177.388 & 358.775 & 357.729 & 359.22 & 359.672 & 0.19989 \\
\hline
\end{tabular}

APTWE, Alpha power transformed Weibull exponential; SE, standard error; -Log L, minus log-likelihood; APTIL, alpha power transformed Weibull inverse Lomax; APTL, alpha power transformed Lindley; APTE. alpha power transformed exponential; AIC, Akaike information Criterion; BIC, Bayesian information criterion; CAIC, corrected Akaike information criterion; HQIC, Hannan-Quinn information criterion; KS, Kolmogorov-Smirnov test statistic

Table 7 Analytical results of the APTWE model and other competing models for the second data set.

\begin{tabular}{|c|c|c|c|c|c|c|c|}
\hline Model & ML Estimates (SE) & $-\log L$ & AIC & BIC & CAIC & HQIC & KS \\
\hline APTWE & $\hat{\alpha}=99.036(100.712) \hat{\beta}=0.078(0.03) \hat{\theta}=0.193(0.069)$ & 269.911 & 545.823 & 544.92 & 546.344 & 548.007 & 0.14042 \\
\hline APTIL & $\hat{\alpha}=8.81(15.855) \hat{a}=0.551(0.281) \hat{b}=23.453(10.978)$ & 291.608 & 589.217 & 588.314 & 589.739 & 591.401 & 0.2677 \\
\hline APTL & $\hat{\alpha}=4.359 \times 10^{-6}\left(6.762 \times 10^{-5}\right) \hat{\gamma}=8.754 \times 10^{-3}\left(6.886 \times 10^{-3}\right)$ & 296.747 & 597.495 & 596.892 & 597.939 & 598.951 & 0.2186 \\
\hline APTE & $\hat{\alpha}=4.822 \times 10^{-8}\left(3.528 \times 10^{-6}\right) \hat{\gamma}=1.361 \times 10^{-3}\left(6.19 \times 10^{-3}\right)$ & 283.583 & 571.167 & 570.565 & 571.611 & 572.623 & 0.19311 \\
\hline
\end{tabular}

APTWE, Alpha power transformed Weibull exponential; SE, standard error; -Log L, minus log-likelihood; APTIL, alpha power transformed Weibull inverse Lomax; APTL, alpha power transformed Lindley; APTE. alpha power transformed exponential; AIC, Akaike information Criterion; BIC, Bayesian information criterion; CAIC, corrected Akaike information criterion; HQIC, Hannan-Quinn information criterion; KS, Kolmogorov-Smirnov test statistic 

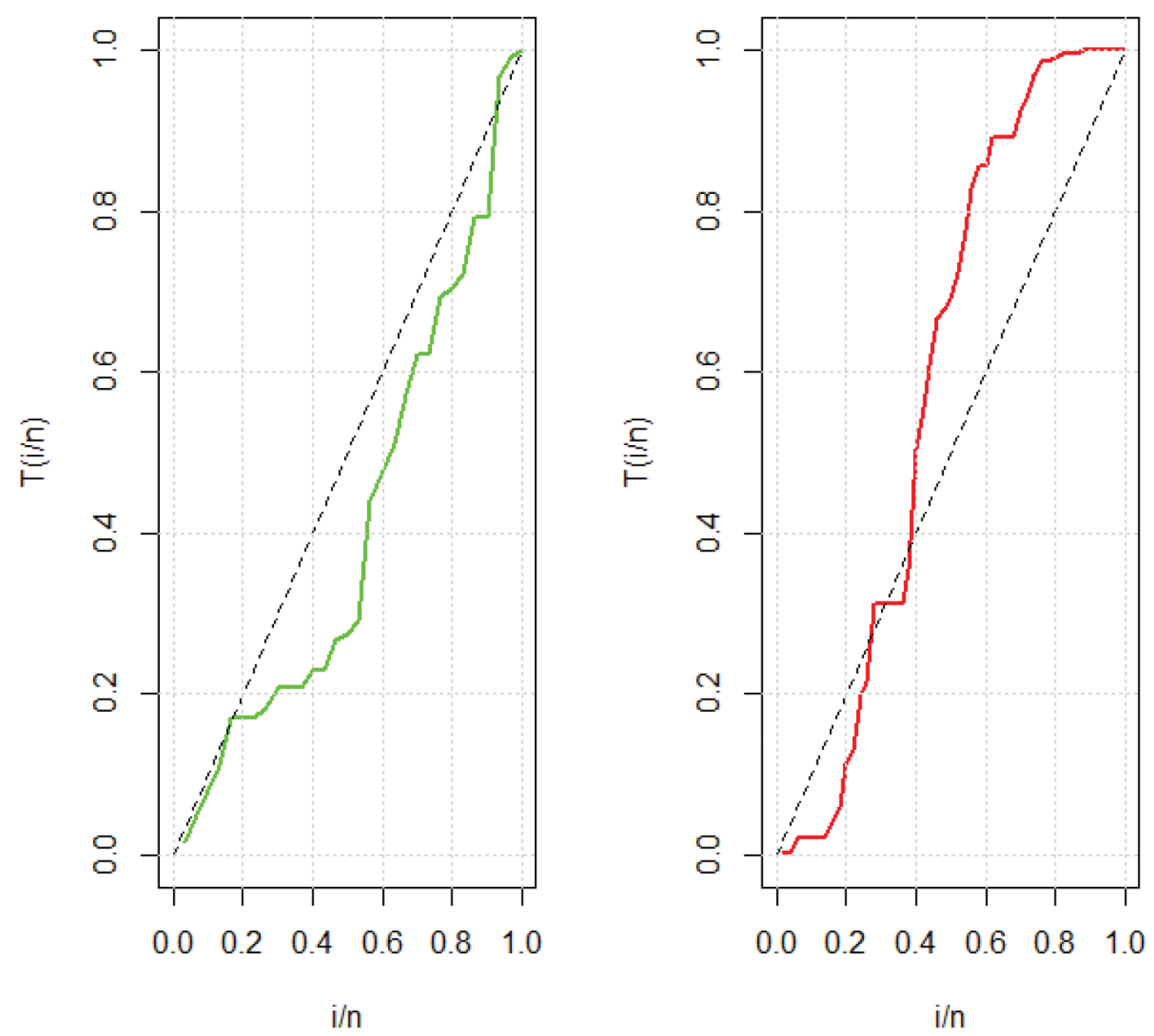

Figure 5 TTT plots of both data sets.
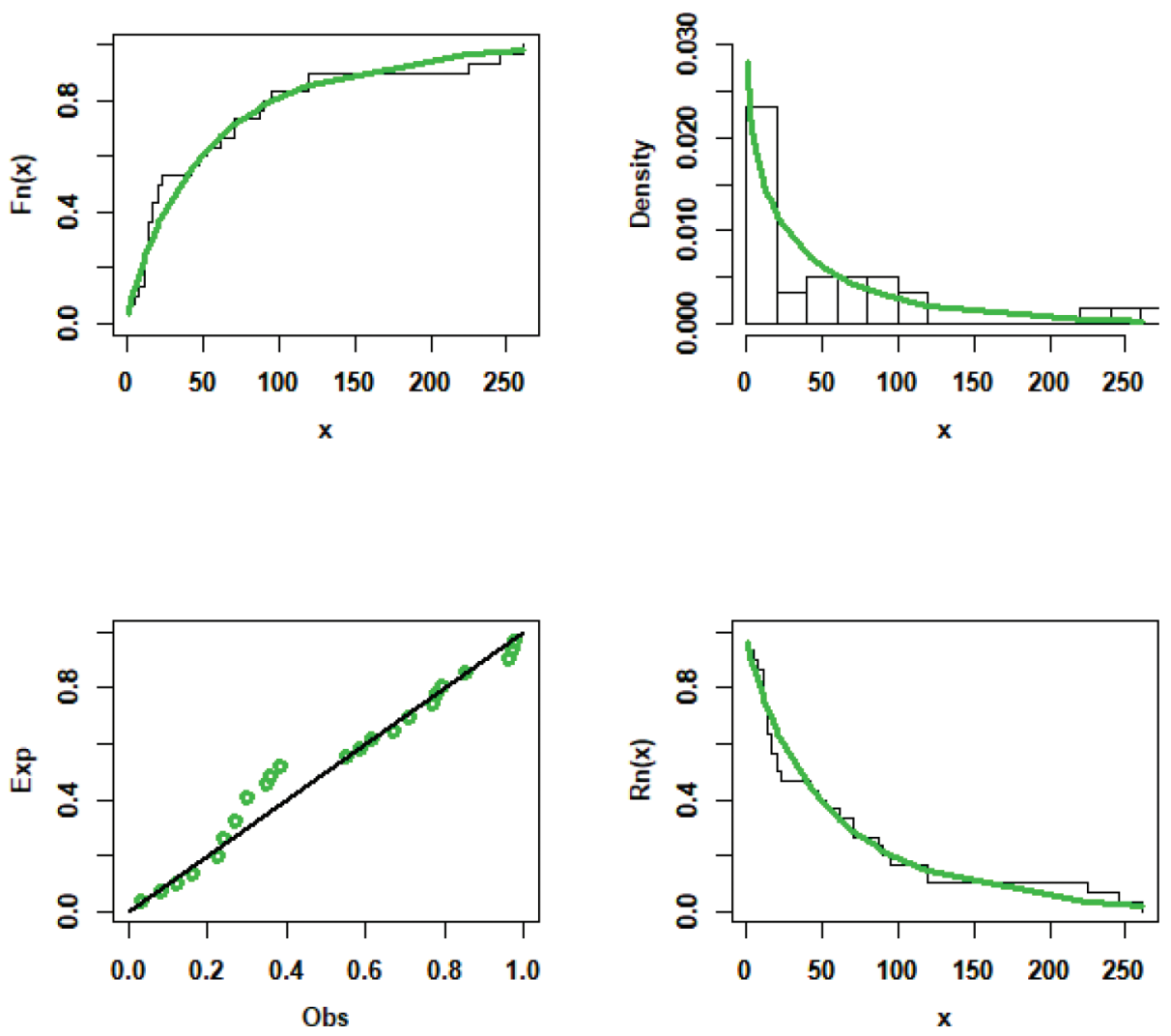

Figure 6 ecdf, epdf, PP plots and esf for the first dataset. 

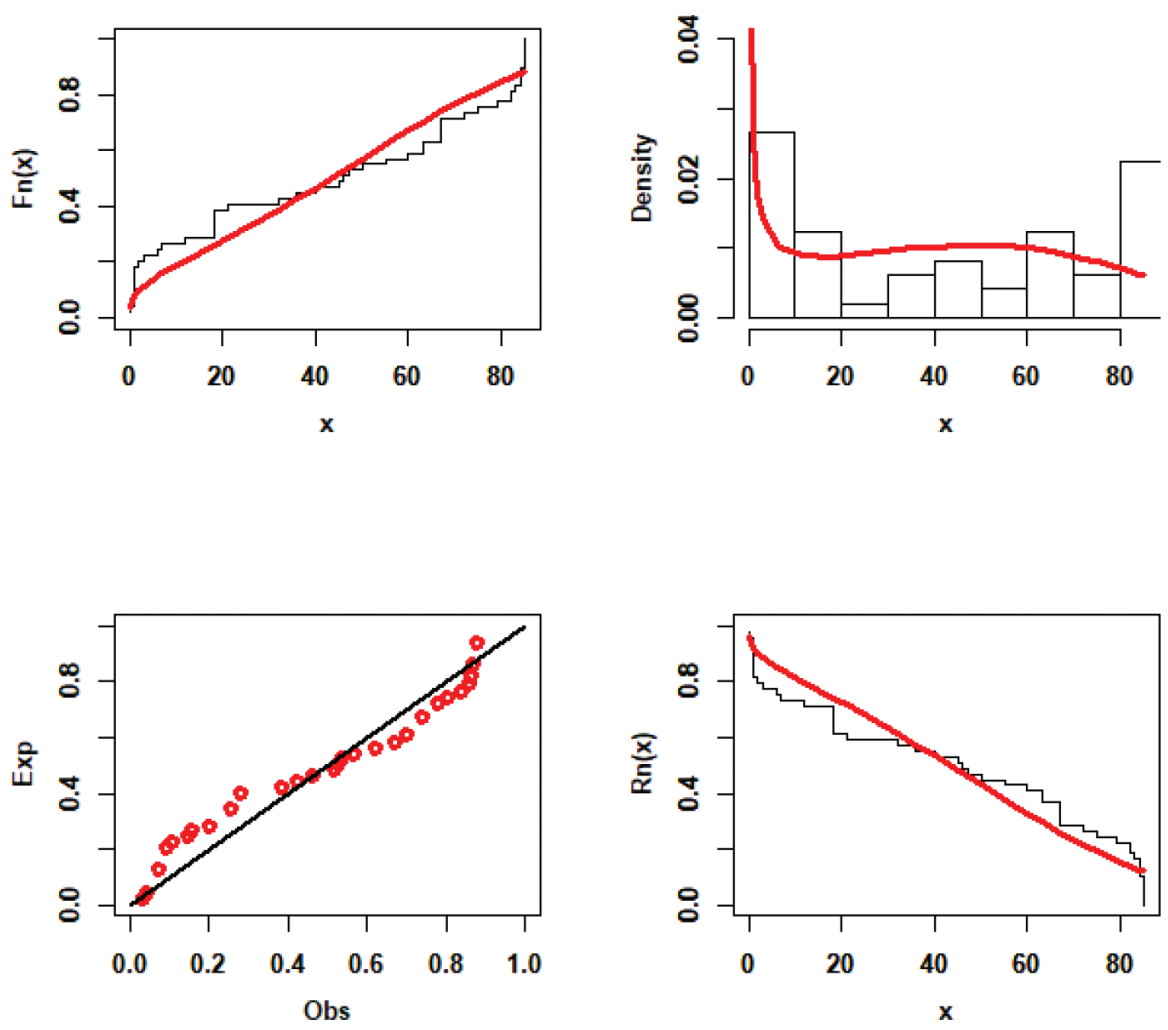

Figure 7 ecdf, epdf, PP plots and esf for the second dataset.

\section{CONCLUSIONS}

In this paper, we developed three special cases (Exponential, Rayleigh and Lindley) of the family of generalized distributions, called APWG family. The density function of the proposed models can have numerous forms: increasing, decreasing, negatively-skewed, positively skewed or symmetrical depend on the values of parameters. Some of useful statistical properties, such as expansion of density function, moments, generating function, incomplete moments, mean deviation, Bonferroni and Lorenz curves are provided. We also discuss the method of maximum likelihood to estimate the model parameters. The performance of the new family of distributions is illustrated by means of a four real data sets. It is observed that the proposed distribution perform well than some competitive distributions. It is expected that this paper will be useful for the users or researchers, those are listed in Section 1.

\section{CONFLICTS OF INTEREST}

The authors declar no conflict of interest.

\section{AUTHORS' CONTRIBUTIONS}

All authors have equally contributed for the manuscript.

\section{ACKNOWLEDGMENTS}

Authors are grateful to anonymous referees for their valuable comments and suggestions, which improved the quality and presentation of the paper greatly. Author, B. M. Golam Kibria wants to dedicate this paper to his very favorite teacher, Late Prof. M Kabir, Department of Statistics, Jahangirnagar University, Bangladesh for his wisdom, constant inspiration during student life and affection that motivated him to achieve this present position.

\section{REFERENCES}


3. A. Marshall, I. Olkin, Biometrika. 84 (1997), 641-652.

4. N. Eugene, C. Lee, F. Famoye, Commun. Stat. Theory Methods. 31 (2002), 497-512.

5. G.M. Cordeiro, M. Alizadeh, M. Tahir, M. Mansoor, M. Bourguignon, G. Hamedani, J. Stat. Comput. Simul. 87 (2017), $908-932$.

6. Z.M. Nofal, A.Z. Afify, H.M. Yousof, G.M. Commun. Stat. Theory Methods. 46 (2017), 4119-4136.

7. F. Gomes, A. Percontini, E. de Brito, M. Ramos, R. Venancio, G. Cordeiro, Austrian J. Stat. 46 (2017), 57-79.

8. Z. Ahmad, M. Elgarhy, N. Abbas, J. Stat. Model. Theory Appl. 1 (2018), 1-16.

9. I. Elbatal, Z. Ahmed, M. Elgarhy, A.M. Almarashi, J. Nonlinear Sci. Appl. 11 (2019), 1099-1112.

10. R.A. Bantan, F. Jamal, C. Chesneau, M. Elgarhy, Entropy. 21 (2019), 1-24.

11. F. Jamal, C. Chesneau, M. Elgarhy, J. Stat. Manag. Syst. 23 (2019), 617-641.

12. R.A. Bantan, F. Jamal, C. Chesneau, M. Elgarhy, Entropy. 21 (2019), 1-22.

13. A.M. Almarashi, F. Jamal, C. Chesneau, M. Elgarhy, F. Jamal, I. Elbatal, Entropy. 12 (2020), 1-21.

14. M. Aldahlan, M. Elgarhy, F. Jamal, C. Chesneau, Entropy. 22 (2020), 1-25.

15. R.A. Bantan, F. Jamal, C. Chesneau, M. Elgarhy, Symmetry. 12 (2020), 1-22.

16. A. Mahadavi, D. Kundu, Commun. Stat. Theory Methods. 46 (2017), 6543-6557.

17. S. Dey, I. Ghosh, D. Kumar, Ann. data Sci. 6 (2015), 623-650.

18. S. Dey, M. Nassar, D. Kumar, J. Comput. Appl. Math. 348 (2019), 130-145.

19. A.S. Hassan, R.E. Mohamd, M. Elgarhy, A. Fayomi, J. Nonlinear Sci. Appl. 12 (2018), 239-251.

20. A.S. Hassan, M. Elgarhy, R. Mohammed, S. Alrajhi, J. Probab. Stat. 2019 (2019), 1-13.

21. S. Ihtisham, A. Khalil, S. Manzoor, S.A. Khan, A. Ali, PLoS One. 14 (2019), 1-15.

22. H. Linhart, W. Zucchini, Model selection. (1986).

23. M.V. Aarset, IEEE Transactions on Reliability, 36 (1987), pp. 106-108.

24. R.A. ZeinEldein, M.A. Haq, S. Hashmi, M. Elsehety, Complexity. 2020 (2020), 1-15. 


\section{APPENDIX}

$$
F_{A P T}(x ; \alpha, \delta)=\left\{\begin{array}{l}
\frac{\alpha^{G(x ; \delta)}-1}{\alpha-1} \text { if } \alpha>0, \alpha \neq 1 \\
G(x ; \delta) \text { if } \alpha=1
\end{array}\right.
$$

and

$$
f_{A P T}(x ; \alpha, \delta)= \begin{cases}\frac{\log (\alpha) \alpha^{G(x ; \delta)} g(x ; \delta)}{\alpha-1} & \text { if } \alpha>0, \alpha \neq 1 \\ g(x ; \delta) & \text { if } \alpha=1\end{cases}
$$

where $\delta$ is the parameters vector for the basline $\operatorname{cdf} G(x ; \delta)$.

$$
F_{W G}(x ; \theta, \delta)=\int_{0}^{\frac{G(x ; \delta)}{\bar{G}(x ; \delta)}} \theta t^{\theta-1} e^{-t^{\theta}} d t=1-\exp \left\{-\left[\frac{G(x ; \delta)}{\bar{G}(x ; \delta)}\right]^{\theta}\right\}
$$

and

$$
f_{W G}(x ; \theta, \delta)=\theta g(x ; \delta) \frac{G(x ; \delta)^{\theta-1}}{\bar{G}(x ; \delta)^{\theta+1}} \exp \left\{-\left[\frac{G(x ; \delta)}{\bar{G}(x ; \delta)}\right]^{\theta}\right\}
$$

respectively, where $g(x ; \delta)$ and $G(x ; \delta)$ are pdf and cdf of any baseline distribution depending on a vector of parameter $\delta$. 